\title{
Evaluating the Health Care the Level of Electronic Health (e-Health) among Healthcare in Developing Countries and It Application in the Management of COVID-19 Pandemic Outbreak
}

\section{Stephen 0* \\ Department of Tele Health Nelson Mandela School of Medicine, University of Kwazulu Natal, South Africa \\ *Corresponding author: Olorunfemi Stephen, Department of TeleHealth Nelson Mandela School of Medicine, University of Kwazulu Natal, South Africa}

Received: January 19, 2021; Accepted: February 10, 2021; Published: February 17, 2021

\begin{abstract}
Context: Healthcare institution in developing countries is in a state of great despair, with the lack of basic health infrastructure and support services, compounded with the ever increasing number of old people , chronic diseases and the manifestation of new communicable disease such as the ongoing Severe Acute Respiratory Syndrome- Coronavirus Disease 2019 (SARS-CoV-2, 2019 or COVID-19) pandemic. The massive healthcare disruption cause by the pandemic with respect to the management modality such as social distance and contact tracing re-affirm the importance of adopting the electronic health system in developing countries. This evolutionary trend in Information and Communication Technology (ICT) with the advancement in eHealth is changing the face of health care system globally by bringing healthcare closer to the people in term of cost, accessibility and planning.
\end{abstract}

Despite the well documented benefits of eHealth, the knowledge and the level of adoption of these technologies in most developing countries such as Lesotho is nothing to ride home about (still remains very low). The management of Covid-19 patients has proven that healthcare is not confined; but a structured global system, with people travelling long distances than ever before.

Objective: The objective of this article therefore, is aimed at evaluating the state and the level of Electronic Health (e-Health) and its adoption in the management of Covid-19 pandemic outbreak, and to also provide the authorities with information on the importance e-Health technology in this 21 century. The paper therefore reveal that, the level of e-Health in developing country like Lesotho, is still very low, due to lack of political commitment in the provision of needed infrastructure support structures.

Keywords: E-health; Cocid-19; Application; Healthcare and developing countries

\section{Background/Introduction}

There is no misgiving that health information technology in particular in the field of electronic health (e-Health), is playing an indispensable role in changing our lives. This technology has ease our access to health information data twenty-four hour a day, with the ability to access information databases used decision support tools and ultimately partaking in the management of our healthcare [1].

The present day healthcare services is now moving toward a new awareness of health information technology globally, therefore the need to transform the healthcare delivery system in developing countries by the implementing and adopting the electronic health system cannot be over emphasis in this $21^{\text {st }}$ century [2]. These technologies via the Mobile Health (m-Health) are playing a major role in the response to the Covid-19 pandemic. These applications are being used to track infected people, issue self-quarantine guidelines, providing latest communication and education to the citizens, to reduce and ease the burden of fake new among the population. In situation where the president of the United State of America give unverified information about the benefit of drinking disinfectant in the management of this infectious disease.

The massive health care system disruptions caused by the corona virus pandemic reiterate, the importance of electronic health system with its efficient patients data management tools. This is because accurate and reliable medical information are crucial for optimizing care, reducing medical errors and improving coordination between providers worldwide. Country with poor electronic healthcare systems is therefore bound to experience, a poorly managed corona virus infection. In addition to these, study has reveal that the level of eHealth in any country is directly align with the economic growth as well as the productivity and the wellbeing of it citizen.

Electronic health therefore is simply using technology to improve the quality of healthcare, and its advances has allowed both patients and medical professionals to gain access to a variety of resources, thereby making healthcare more cost efficient. Most significantly it
Austin Intern Med - Volume 5 Issue 1 - 2021

Submit your Manuscript | www.austinpublishinggroup.com

Stephen. (C) All rights are reserved
Citation: Stephen O. Evaluating the Health Care the Level of Electronic Health (e-Health) among Healthcare in Developing Countries and It Application in the Management of COVID-19 Pandemic Outbreak. Austin Intern Med. 2021; 5(1): 1051. 
has empowers patients to take more active part in their treatment, thus allowing them to gain a deeper understanding of their conditions and how to effectively work with health care managers.

Looking at "e" in e-Health holistically, one will simply assume it simply represents the word "electronic." Though "electronic" is certainly one aspect, the complete definition actually encompasses a variety of other "e's," representing the true meaning of this concept out line bellow as Efficiency, Enhancing Quality, Evidence Based, Empowerment, Encouragement, Education, Enabling, Extending and Ethics and Equity [3].

The introduction of ICT in healthcare, predominantly in the area of mobile phones and also compact computer also referred to as PDA (personal digital assistant). This system was originally built to function as a personal scheduling assistant are now playing a considerable role in improving health care in multiple rural settings. Electronic health is also playing a crucial role in supporting healthcare worker in rural setting, in performing clinician duties where there are no doctors thereby providing a huge potential for health care organizations to deliver a quality, cost-effective care to geographically disadvantage populations in low and middle-income countries. The European Commission define e-Health: as the use of modern information and communication technologies to meet needs of citizens, patients, healthcare professionals, healthcare providers, as well as policy makers." Combining both definitions we can say it is a technology tool used specifically as an electronic communication within healthcare environments and institutions [4].

There are numerous names accompanying the abbreviation describing the use of computer systems or ICTs in healthcare delivery. Some of the names could be described as follow: Electronic Medical Record (EMR), Electronic Patient Record (EPR), Computerized Medical Record (CMR), Computer-Based Patient Record (CPR), and Electronic Health Record (EHR). The above listed terms often used interwoven to mean the same thing, but there are some minor differences in the meanings depending on the defining authority [5]. In simpler term EMR is simply a systematic collection of patient or population data electronically and this information is stored in a digital format. EMR is the electronic version of the paper-based record, a computer-based system for storing, organizing, and retrieving information about patients.

Statement of problem: The human toll from this global Covid-19 pandemic outbreak is in orders of ongoing magnitude, that is becoming larger than the health-care system and the economy of many developing countries will be able to cope. The healthcare systems at the moment are facing unprecedented pressure following this pandemic outbreak. Looking at the effect of this pandemic is having on the health care system of the United State of America, France, Italy or the United Kingdom who are struggling to curtail this pandemic, one can then imagine what will happen to most developing countries with little or meaningful supply of ventilators, a crucial resuscitating tool for treating serious cases of COVID-19 patients. Taken for example Nigeria is reported to have fever than 500 ventilator in total a country with a population was estimated to be about 206 million individuals., while the Central African Republic with about 5 million may have no more than three ventilator what a pathetic situation.
Therefore, some healthcare in developing world can be said to be is in a state of great despair and needs; this is because healthcare system lack the basic required infrastructure and support services. According to the World Health Organization, developing countries like Lesotho are facing formidable public health challenges, including low coverage of essential public health services. The country is with 372 healthcare facilities scattered all over the country, still most of these health institution operate on parallel paper system with little or no electronic health reporting systems, making it difficult to properly follow program achievements or detect potential impediment to the system. What will be the outcome in the event of massive Covid-19 infection your answer is as good mine?

The present outbreak of Covid-19 in many of these developing countries could be a sign that, the crisis may barely have begun, and the human toll from this global pandemic outbreak would be orders of magnitude larger than the health care system and the economy can cope with globally. The management of this infectious disease using modernized technology like the e Health cannot be overemphasis. It will therefore be a big mistake for developing countries to depend continuously on developed countries for assistance because some of these countries are struggling to keep their healthcare system afloat, for example vaccine (BNT162b2 Pfizer and the mRNA-1273 Moderna vaccine), distribution.

\section{The Level of Electronic Health in Developing Countries Using Lesotho as a Case Note}

Despite the well-documented benefits of eHealth technology globally, the knowledge and the level of these technologies in Lesotho is still remains very low. The up to date patient information system in Lesotho operates in silos this is because every system available has the capacities for full data entry, storage and processing of statistical or operation reports with the installed software [6]. As highlighted above, all the health data or services are compiled manually and later computerized where applicable and can be viewed. In other word, most of the system available in the country can be said to be a NonIntegrated Clinical and Data Systems [7].

The DHIS2 system has since been rolled out centrally and across all of Lesotho's ten districts, and now covers all 333 health facilities throughout the country. It houses over 40 million records and 33 datasets related to key technical areas, including HIV care and treatment [8]. What part would this play in the management of Covid-19 is yet to be determined, since the system is not holistically own by the Government.

\section{The Importance of Electronic Healthcare Application in Developing Countries}

The efficient adoption and implementation of e-Health in many developed counties has revolutionized the health care systems in the $21^{\text {st }}$ century [9]. Some of the e Health areas include: Electronic Health Records (EHR), Electronic Medical Records (EMR), Personal Health Records (PHR), and Health Information Systems (HIS), telemedicine, e-prescription, Remote Patient Monitoring (RPM) and diagnosis [10].

The advancement of this technology has made it possible for it to replace many functions of the traditional paper chart, which has led to significant advances in patient care [11]. Thus, e-health 
is fast becoming a reality in both developed and few developing countries and holds great promise in improving global access to healthcare services and health informatics as a whole. Looking at it critically electronic health has presented healthcare institution with opportunity this has presented an opportunity that enhances disease prevention by extending health interventions beyond the reach of traditional care, an approach commonly referred to as mobile Health. The Mobile Health (m-Health) field has emerged as a sub-segment of e-health, and it's, applications include the use of mobile devices in collecting community and clinical health data, delivery of healthcare information to practitioners, researchers and patients. It facilitate and help in supporting key processes such as improved access to health services, disease diagnosis and treatment, patient monitoring and provision of some client-centres health information.

\section{How Prepare are the Developing World in} the Adoption of e-Health and Its Utilization in the Management of the Corona Virus Pandemic

Covid-19 has devastated the health care systems globally with an estimated mortality till date Monday, 18 January 2021 2,045,418 and with about 95,833,79520 Cases [12]. In some developing countries like Liberia, according to expert, there are just about three ventilators for the entire population, the simple fact is that most of this countries lack the capacity to mitigate its effects of corona virus in the population.

The term 'healthcare information infrastructure' implies a coherent and comprehensive set of information systems and services, necessary and sufficient to support the goals of healthcare services and their governance. The adoption of an e-health with poor planning will do any country more harm than good in future. It is unfortunate that some great health intervention or programmers with proven benefit in developed countries, such as the electronic health application technology have sometime fail to get pass the pilot state in most developing countries. This is because of the absences of the required infrastructure, poor environmental assessment and the required supports system. It is well established that three quarters of Computerized Information Systems (CIS) fail, an example is the Electronic Medical Record (EMR) project costing the tax payers about 134 milloin of Rand in Limpopo Province in South Africa by the International Business Machine (IBM) fail woefully the question is why? [13]. The design and implementation of e-Health Information Technology projects often skip the stage such as through environmental assessment without understanding what the end users of the technology need and how important this technology can best be used to address these crucial areas [13].

\section{Electronic Health Context}

E-Health main objective is aims at delivering health services and information through the Internet and other related technologies [14]. Globally its adoption has been low in some developed countries, but much lower in developing countries. Most of this developing countries are confronted with other additional challenges of insufficient health infrastructure, the deficient of technical expertise and the lack of support services $[15,16]$.

Information technology infrastructures is an evolving phenomena that are dynamically and incessantly defined and re-defined within their use in the society, rather than simply looking at the planned consequences of adopting such technology, or the result of planned actions by users and organizations.

E-Health technology applications are well known tools innovative that is playing significant role in the improvement of quality of life and quality healthcare delivery in most health institution both in developed and developing countries. It is unfortunate that despite this positive benefit, studies carried out in most developing countries indicate that the rate of implementation and adoption of this advancement in e health is either low or non-existence. Other positive benefit attributed to e-health is that it allows health organizations and institution to streamline many of their activities leading to a more efficient and cost-effective patients care. It also reduced errors and missing files, reduced work task, reduced expenditure on paper logistics and the retrieval of absconded bills. In retrospective the management of Covid-19 patients has demonstrated that healthcare is not local; it is an interconnected global system, with people travelling long distances than ever before, it is therefore immature to look at regions of the world in isolation from each other and try to manage public health in parallel. To effectively deal with this present situation, the scope of health information exchange efforts such as the electronic should not be limited to the country or community in question but instead should be done at least nationally, if not internationally. It was reveal by the International Telecommunications Union data; that only $31 \%$ of the developing world population use internet, from this $16 \%$ in Africa, while $90 \%$ of households in developing world has no access to Internet, countries like Germany, England and the Netherlands are well ahead in the area of EMR implementation [16]. Some challenges to the implementation and adoption of this system in some developing counties include; poor network connections and unstable power supply as experience in Nigeria.

Studies conducted in few developing countries notice that the use of e-health's system in healthcare delivery has led to a better access into healthcare for both patients in the remote and inaccessible areas [17]. In addition health information technology has leads to timely health policies and planning, improvement in the general health status of the population, as well as, serving as a vital element for individual health facilities in managing and improving healthcare delivery [18]. In order word software engineers and all the stakeholders should focus on the roles of health care providers at all levels and how information and communication technology can be an enabler to their jobs or service with adequate environmental assessment and with the support of the local human factors (electronic health specialist) in the countries [19].

It is important to note that many study conducted emphasis that to successfully implement and adopt an e-Health system, health care providers must accept the technological systems and the potential disruptions and changes that follow the adoption of this system [20]. It is unfortunate that some health care providers still perceived the introduction of e-health technology into the system, as interfering with clinical workflow, reducing productivity, and introducing disruptive changes to the workplace with such observed tendency is much more serious in developing countries where computer illiteracy is very poor [21]. This is supported by a the pilot e-health implementation phase conducted in Ethiopia, it was noted that user resistance was reported 
to be the primary hindering factor to its successful adoption of the technology [22].

Electronic healths via the mobile health are playing a big role in the response to the Covid-19 pandemic. All these application are being used to track infected people, issue self-quarantine guidelines, providing latest communication and education to the citizens to reduced and ease the burden of fake new among the population. In order word electronic health technology has come to the rescue of healthcare staffs in the diagnoses of those infected, affected, identifying hotspots and getting essential updates. Thereby helping to significantly slow the rate of transmission of this virus, and support countries to emerge from lockdowns safely. Numerous applications are presently in used in Italy, India, South Africa, Ghana and Angola, while other is undergoing development worldwide.

\section{Some example of e-health applications includes:}

- Aarogya Setu Application used in India this Covid-19 tracking application referred to as Aarogya Setu became the world's fastest growing application. Aarogya Setu referred to in India as (The bridge for liberation from disease) this is an Indian open-source cross-platform CoVID-19 "Contact tracing, Syndromic mapping and Self-assessment" digital services [23].

\section{- COVID Community Alert used in Italy}

Another well-known application is the COVID Community Alert is an Open-Source service that allows the monitoring of interactions among mobile devices in a totally anonymous fashion. It helps in reducing the spread of the virus by alerting people at risk and sending them a clear set of instructions to help them to reduce contagion and receive treatment as soon as possible [24].

- United Kingdom: COVID Symptom Study, formerly Covid Symptom Tracker and NHS COVID-19, How We Feel (selfdiagnostic) Private Kit: Safe Paths (contact tracing) Covid Watch (digital contact tracing and alerting, coEpiself-reporting NOVID contact tracing [25].

- United States of America (USA): COVID-19,Screening, self-diagnostic and Tool Covid Safe application self-diagnostic, contact tracing Alipay Health Code" for contact tracing

- Italy: Covid Community Alert (Covid application for citizens (proximity tracing and exposure notification, optional GPS location sharing), CovidDoc for doctors (scan patient QR code, log patient health status), web dashboard for epidemiologists (set parameters that trigger notifications) [26].

- $\quad$ South Africa: Covi-ID for contact tracing, health credential management system [27].

- Ghana: GH COVID-19 Tracker App [28].

- Angola: COVID-19 AO used for self-diagnostic, information and quarantine enforcement [28].

- Lesotho: But here in Lesotho the information is still mainly by cell phone and what- up group application and the DHIS application no specific application (nil application)

Some Crucial Role Play by the Electronic Health and Lesson Learnt in Managing the Pandemic

The disparity or inequalities in our health care system need to be critically examine, based on the fact that creative partnerships with digital economy can create a better world for all.

The use of Tele-health has allowed thousands of patients to be screened and treated at home where necessary thereby, giving hospitals time to prepare and deal with essential and emergency cases. This management modality fit in with the present slogan of social distance one of the cardinal point in the management of Covid-19. Adequate monitoring and treatment of these patients remotely, helps in reducing hospital-related costs and improve quality of life, patients therefore have a greater independence in participating in managing their healthcare.

\section{Other importance lesson includes:}

- $\quad$ Serving as a reliable surveillance System, the need to have an effective electronic surveillance system, in the management of health disease is fundamental in conjunction with an accurate and timely data collection from every part of country. The data collection process are made more easy, efficient and reliable when conducted via smart phones, Personal Digital Assistance, or mobile phones rather than paper-based system [29].

- Strengthening of healthcare capacity with Electronic prescription

The health care institutions in developing countries are face with poor health infrastructure and support services. The promise of digital medicine of the future will provide an unparalleled opportunity to address the social determinants of health.

The acute shortage of healthcare workers is a major challenge facing the health sectors. Therefore training new cadres of health professionals and empowering them with basic ICT training is vital [29].

\section{- Improving healthcare infrastructure and support} services:

The continuous lack of health workers present a huge dilemma, so the shifting of medical staff from their usual post to Covid-19 care has exacerbates the shortages in other areas; these shortages has led to the badly managed usual activity in the system. The continuous losing of healthcare workers to Covid-19 pandemic is having a major impact on health provision, in an already poor staff health facility. A well-integrated e-health system wills assist in tracking Covid-19 suspect and contact tracing system. It is therefore essential that health information management (HIM) professionals ensure COVID-19 documentation, data capture, data analysis and reporting, as well as coding are accurate and reliable to support clinical healthcare.

\section{Conclusion}

One of the major problems at the present is how to improve the health care infrastructure to ensure adequate delivery of an effective health care system. The main rationale therefore, is to be cautions and do it right by addressing some fundamental concerns that are presently undermining the ability to provide the an efficient healthcare system. The dilapidated states of health care system in developing world re affirm the lack of capacity to address the corona virus pandemic, since some countries cannot boast of adequate supplies of basic health equipment such ventilators, a crucial tool required in the management 
of severe cases of COVID-19. The mHealth applications is helping significantly in slowing the rate of transmission of this virus, thereby supporting countries to emerge from lockdowns safely, as restrictions are gradually easing down globally. The basic e-Health skill and experience required in our health care system are weak or missing and the technology that allows scalability and inter-operability with other existing systems are nonexistence. All developing countries needs to focus on health systems strengthening, the continuous loss of health care workers through migration to developed countries like Europe for a better life, will continues to deplete the number of health staff available in the system. Although our present paper-based systems can provide a partial solution, but the information transmission is slow and prone to errors.

\section{References}

1. Health Information Systems in Developing Countries, a Landscape Analysis Vital Wave Consulting HIS. 2009.

2. World Telecommunication/ICT Development Report 2010. International Telecommunications Union, Geneva. 2010.

3. World Health Organization (WHO), e Health resolution 2005. 2011.

4. European Commission, Ministerial Declaration. Brussels. 2011.

5. Nohr C. Evaluation of electronic health record systems. International Medica Informatics Association, Yearbook of Medical Informatics. 2006: 107-113.

6. Government of Lesotho. 'Guidelines to prevent Mother to Child Transmission of HIV'. 2016.

7. UNDP. Human Development Report. Health Action in Crises Lesotho. 2002.

8. In Lesotho. New Health Information System Provides Streamlined. Integrated Data Across Health Programs. 2015.

9. Ghias US, Mansoor NB, Mehwish I, Muhammad IQ. Khalid Z. Implementation of Technology Acceptance Model in E-Learning Environment in Rural and Urban areas of Pakistan. World Applied Sciences Journal. 2013; 27: 14951507.

10. Busagala LSP. Perceptions and Adoption of Information and Communication Technology for Healthcare Services in Tanzania. International Journal of Computing and ICT Research. 2013; 7: 12-21.

11. Institute of Medicine. Committee on Improving the Patient Record. The Computer-based Patient Record: An Essential Technology for Health Care. Rev edition. Dick RS, Steen EB, Detmer DE, ediotrs. In: Washington, DC National Academy Press. 1997.

12. Worldometer. COVID-19 Coronavirus Pandemic. 2021.

13. Littlejohns P, Wyatt J, Garvican L. Evaluating computerised health information systems: Hard Lessons Still to Be Learnt. BMJ (Clinical research edition). 2003; 326: 860-863.
14. Mugo DM. 'Determinants of electronic health in developing countries'. International Journal of Arts and Commerc. 2014; 3: 49-60.

15. Lam MK, Hines M, Lowe R, Nagarajan S, Keep M, Penman M, et al. 'Preparedness for eHealth: Health sciences students' knowledge, skills, and confidence'. Journal of Information Technology Education: Research. 2016; 15: 305-334.

16. Gillwald A, Mothobi O. After Access 2018: A Demand-Side View of Mobile Internet from 10 African Countries. 2019.

17. Yusif S, Soar J. Preparedness for e-Health in developing countries: the case of Ghana. Journal of Health Informatics in Developing Countries. 2014

18. Khan SZ, Shahid Z, Hedstrom K, Andersson A. Hopes and Fears in Implementation of Electronic Health Records in Bangladesh. The Electronic Journal of Information Systems in Developing Countries. 2012; 54

19. Teviu EAA, Aikins M, Abdulia TI, Sackey S, Boni P, Afari E, et al. Improving Medical Records Filing in a Municipal Hospital in Ghana. Ghana Medical Journal. 2012; 46: 136-141.

20. Senafekesh $\mathrm{Y}$, Tesfahun $\mathrm{H}$, Mulusew A, Binyam B. "Health Professionals? readiness to implement electronic medical record system at three hospitals in Ethiopia: a cross sectional study". BMC Medical Informatics and Decision Making. 2014; 14: 115.

21. Gamm LD, Barsukiewicz KC, Dansky KH. "Pre- and post-control model research on end-users' satisfaction with an electronic medical record: preliminary results. In Proceedings of the AMIA Symposium". in American Medical Informatics Association, Pennsylvania, United States. 1988.

22. Li J, Talaei-Khoei A, Seale H, Ray PMC. "Health Care Provider Adoption of e-Health: Systematic Literature Review". Interactive Journal of Medical Research. 2013.

23. Govt launches 'Aarogya Setu'. a coronavirus tracker app: All you need to know". Livemint. 2020.

24. Covid Community Alert. world-level Open-source anonymous protocol. 2020.

25. O'Malley K. "16 Apps Everyone Should Have On Their Phone During Coronavirus". ELLE. 2020.

26. Lorenz CK, Delpriori S, Di Francesco GM, Maldini R, Paolini BD, Bogliolo A. Alessandro "Digital Ariadne: Citizen Empowerment for Epidemic Control". 2020.

27. Moche T. "COVID Tech Part I: Government, UCT partner for tracing app". Sabcnews. 2020.

28. "Why Ghana govment do virtual concert take launch COVID-19 app way people no fit download?". BBC News Pidgin (in Pidgin). 2020.

29. Hanseth O. From systems and tools to networks and infrastructures - from design to cultivation. Towards a theory of ICT solutions and its design methodology implications. Unpublished manuscript. 2020. 